\title{
Imageur diamant et nitrures pour l'observation UV du soleil
}

\author{
J.-F. Hochedez, T. Appourchaux ${ }^{1}$, A. Belsky ${ }^{2}$, M.C. Castex ${ }^{3}$, A. Deneuville ${ }^{4}$, \\ P. Dhez ${ }^{5}$, B. Fleck ${ }^{6}$, O. Hainaut ${ }^{7}$, M. Idir $^{8}$, J.-P. Kleider ${ }^{9}$, P. Lemaire ${ }^{7}$, E. Monroy ${ }^{10}$, \\ E. Munoz ${ }^{11}$, P. Muret ${ }^{4}$, M. Nesladek ${ }^{12}$, F. Omnes ${ }^{13}$, J.-L. Pau ${ }^{11}$, A. Peacock ${ }^{1}$, \\ U. Schühle ${ }^{14}$ et C. Van Hoof ${ }^{15}$
}

Royal Observatory of Belgium, Solar Physics Department, Circular Avenue 3, 1180 Bruxelles, Belgique

${ }^{1}$ ESA-ESTEC, Solar System Division, Noordwijk, Pays-Bas

${ }^{2}$ CELIA, Bordeaux, France

${ }^{3}$ LPL, CNRS, Villetaneuse, France

${ }^{4}$ LEPES, CNRS, Grenoble, France

${ }^{5}$ LIXAM, Orsay, France

${ }^{6}$ ESA, Solar System Division, Goddard Space Flight Center, U.S.A.

${ }^{7}$ IAS, Orsay, France

${ }^{8}$ LURE SOLEIL, Orsay, France

${ }^{9}$ LGEP, Gif-sur-Yvette, France

${ }^{10} \mathrm{CEA}$, Grenoble, France

${ }^{11}$ DIE UPM, Madrid, Espagne

12 IMO, Diepenbeek, Belgique

${ }^{13}$ CHREA, Valbonne, France

${ }^{14}$ MPAE, Katlenburg-Lindau, Allemagne

${ }^{15}$ IMEC, Leuven, Belgique

Résumé : BOLD (Blind to the Optical Light Detectors) est un projet regroupant plusieurs laboratoires, instituts, et industriels européens ; il est dédié aux développements et aux tests de nouveaux détecteurs pour l'observation du Soleil dans l'ultraviolet. Notre investigation repose sur les propriétés des solides à large bande interdite, tels que le diamant ou les nitrures d'aluminium et de gallium (AlGaN). L'initiative est proposée en vue des instruments UV du futur Orbiteur Solaire de l'Agence Spatiale Européenne (ESA), mais plusieurs autres applications scientifiques ou industrielles en bénéficieront. L'insensibilité au visible (solar-blindness) des nouveaux senseurs sera très avantageuse. Elle permettra une plus grande sensibilité des instruments, et donc plus de résolution spatiale, et une plus haute cadence. Une technologie fondée sur le diamant ou sur les AlGaN plutôt que sur le silicium autorisera aussi une résistance accrue aux rayonnements ionisants (rad-hardness), et donc la stabilité indispensable aux conditions extrêmes que connaîtra l'Orbiteur Solaire. Nous présentons ensuite quelques résultats de campagnes de mesures entreprises dans le cadre du projet BOLD, et décrivons le programme envisagé pour obtenir en quelques années des caméras UV très innovantes.

\section{INTRODUCTION}

La détection de l'ultraviolet se heurte à plusieurs difficultés fondamentales, principalement liées au caractère superficiel de l'interaction de ces photons avec l'état solide. Par exemple, la fragilité notoire des senseurs UV envers la contamination par les polluants découle du fait que l'énergie électromagnétique est déposée dans un faible volume, polymérisant ainsi les éventuelles chaînes carbonées en présence. Par suite, les photons UV n'atteignent plus le volume sensible du détecteur dont le rendement est par conséquent réduit. La physique du solide de l'ultraviolet du vide (VUV) et de l'extrême UV (EUV) se produit en surface. Pour sensibiliser les imageurs traditionnels tels que les CCDs à ces gammes de longueur d'onde, il a fallu éclairer leur face «arrière» pour éviter l'absorption par les électrodes de la face «avant». Il a aussi fallu traiter la surface sensible de façon à réduire l'épaisseur de l'oxyde naturel gênant, et à éloigner des interfaces les photoélectrons constituant le signal. En quelques décades, les avancées technologiques ont été remarquables [1], et nous leur devons entre autres les observations de la couronne solaire par la sonde spatiale SoHO, une mission de collaboration internationale ESA - NASA. Cependant, 
les détecteurs UV, et notamment les imageurs, procèdent de technologies généralement conçues pour le visible. Or l'emploi du silicium, dicté par la maîtrise qu'en a l'industrie du semiconducteur, s'avère peu optimal quand il s'agit de mesurer de faibles signaux UV au sein d'un flux visible élevé et d'un environnement sévère de particules ionisantes. Le refroidissement du détecteur -nécessaire à un faible courant d'obscurité- le transforme en piège froid où se fixent les particules d'hydrocarbures qui se polymérisent alors sous l'action de l'UV. La formation d'une couche noire et opaque est irréversible. Refroidir est en outre coûteux et malaisé dans le spatial. Les défauts cristallins créés dans l'oxyde et dans le silicium par les particules ionisantes, mais également par l'UV, sont un second facteur, partiellement réversible, de perte de rendement quantique (QE). De plus, les rayonnements indésirables produisent des artefacts difficiles, voire impossible à distinguer du signal étudié. Enfin, le caractère panchromatique du silicium sensible dès $0.1 \mathrm{~nm}$ et jusqu'à $1 \mu \mathrm{m}$, oblige à interposer des filtres destinés à rejeter les gammes spectrales indésirables, tel le visible. Mais ils atténuent considérablement l'UV ; et leur fragilité (films minces métalliques) ou leur instabilité (filtres interférentiels) constituent un écueil supplémentaire. Les considérations précédentes varient quantitativement avec chaque gamme de l'UV, mais en VUV, les traitements de surface ne suffisent plus. Il faut insérer des galettes de micro-canaux couplées par fibres optiques à l'imageur, et les problèmes afférents à ces derniers s'additionnent à la liste précédente. Une haute tension doit leur être appliquée, ce qui est un problème en soi dans le spatial. Il faut de surcroît augmenter cette tension pour compenser l'usure de la galette. Etalonner un tel système est impossible. Il apparaît ainsi clairement qu'une bonne partie des limitations éprouvées actuellement par les dispositifs imageurs dans l'ultraviolet provient du matériau où le signal électronique est créé.

\section{LES BESOINS EN IMAGEURS UV POUR L'OBSERVATION SOLAIRE}

Les futurs programmes d'observation de l'atmosphère solaire exigent des imageurs UV surpassant l'état de l'art actuel. Pour mieux comprendre la couronne, et pour espérer prévoir ses éruptions qui ont parfois un impact nocif sur notre planète, il est impératif d'améliorer les observations et plus spécialement leur résolution spatiale. Le champ magnétique structure très finement le plasma coronal et donne lieu à une diversité de manifestations plus ou moins transitoires de la magnéto hydrodynamique (MHD). Bien que la boucle magnétique soit la brique élémentaire, elle n'est pas encore bien comprise. Des sous-structures filamenteuses lui sont supposées, et ceci est représentatif de toutes les autres observables (figure 1.).
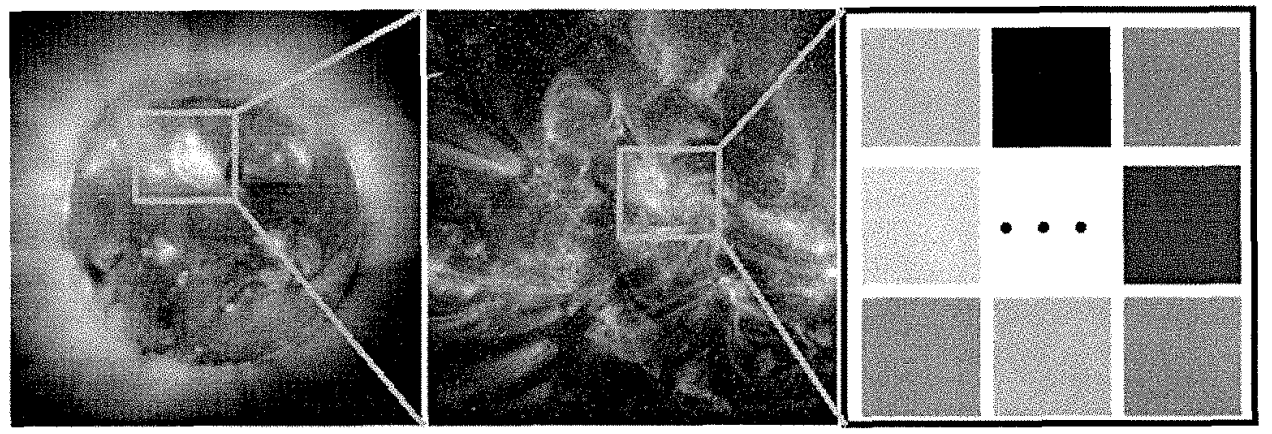

Figure 1. Une nouvelle augmentation de la résolution spatiale est primordiale pour l'exploration et la modélisation des structures dynamiques de la chromosphère à la couronne solaire. L'image de gauche a été réalisée par le télescope EIT-SoHO dans la raie du Fe XV à $284 \AA$. Y sont visibles l'ombre en projection des grilles supportant les filtres en aluminium et la perte de rendement des zones fortement exposées comme le limbe. L'image centrale fut enregistrée le même jour dans les raies du Fe IX/X à $171 \AA$ par TRACE, le télescope solaire EUV disposant actuellement de la meilleure résolution. Incidemment, les impacts de nombreux rayons cosmiques y sont perceptibles.

Rapportée à la surface solaire, la dimension du pixel des images qu'envoie le télescope TRACE de la NASA est d'environ $350 \mathrm{~km}$. Il est admis qu'au moins un ordre de grandeur linéaire -soit deux ordres en surface- sont nécessaires pour faire progresser les modèles physiques. Or, plus les phénomènes sont petits, plus vite ils évoluent. Par extrapolation des observations présentes, il faut s'attendre à exposer environ $0.1 \mathrm{~s}$ pour un pixel de $35 \mathrm{~km}$ de coté, ce qui revient à diviser le temps de pose par cent, 
relativement à TRACE. Il faudrait de ce fait augmenter la surface effective de nos télescopes de quatre ordres de grandeur $[2,3]$ ! Avec la mission Solar Orbiter (S.O.) décidée par l'ESA pour un lancement en 2011, une partie de la solution proviendra de l'orbite qui amènera la sonde à seulement 40 rayons solaires de la surface, soit cinq fois plus près du Soleil que ne l'est la Terre. Un facteur 25 est gagné de la sorte, mais c'est encore insuffisant, et le coût à payer est un environnement radiatif accru. Il est de ce fait crucial de trouver des solutions d'abord conceptuelles puis technologiques, répondant à l'enjeu de l'Orbiteur Solaire. Un détecteur insensible au visible permettrait d'économiser certains filtres, augmentant la surface effective d'un facteur trois à vingt à chaque suppression. Un senseur basé sur un matériau à large bande interdite (WBGM) est susceptible d'offrir les caractères « solar-blind » et « rad-hard» requis. Ce sont les motivations initiales du projet BOLD. De tels détecteurs UV en diamant et en AlGaN existent et sont industrialisés, mais ils ne sont pas imageurs. Notre programme de développement prend en compte les contraintes spécifiques à S.O. énoncées ci-dessus, et se propose de démontrer en 2 ans environ la faisabilité d'imageurs UV de grand format basés sur un matériau à large bande interdite. Ce succès conditionne le maintien de l'option WBGM sur S.O., et préside aux orientations techniques de BOLD. Nous avons formulé en ce sens une proposition de R\&D qui a récemment été approuvée par l'ESA.

\section{NOUVEAUX MATERIAUX ET NOUVEAUX CONCEPTS}

Pour atteindre prochainement les très grands formats $(4000 \times 4000)$ voulus pour la mission S.O., une solution hybride a été adoptée $[3,4]$. Une membrane photo-active convertira les photons UV en signal électronique tout en restant très inerte au visible et aux radiations ionisantes. Un circuit de lecture CMOS lui est hybridé (figure 2a). Par rapport au CCD, la technologie CMOS apporte ici la souplesse des fonctionnalités du pixel, une plus grande tolérance aux radiations, une lecture très rapide et non destructive, etc. Les très grands formats n'ont pas encore été démontrés, surtout en rapport à l'hybridation, et c'est un but réalisable que le projet BOLD se donne. Des pellicules aux dimensions appropriées seront en contact avec la matrice de lecture par des billes d'indium (technologie «Flip-Chip », figure 2). Ce principe sera d'abord démontré pour des dimensions réduites avant d'être porté aux formats requis. Cette approche permet de sérier les éventuelles difficultés pour démontrer dans le temps imparti la faisabilité du dispositif recherché. La méthode choisie impose aussi un feedback continu des tests expérimentaux sur la modélisation physique et le procédé industriel. Il sera notamment important de mesurer la sensibilité des prototypes depuis les $X$ jusqu'au visible pour être en mesure d'optimiser certaines propriétés plutôt que d'autres. Le compromis entre le niveau de saturation, la taille du pixel, le format d'image et la surface de la membrane active n'est qu'un exemple. L'évaluation rapide des profits et déficits relatifs aux technologies concurrentes constitue le second motif favorable à une évaluation rapide des performances en fonction de la longueur d'onde.
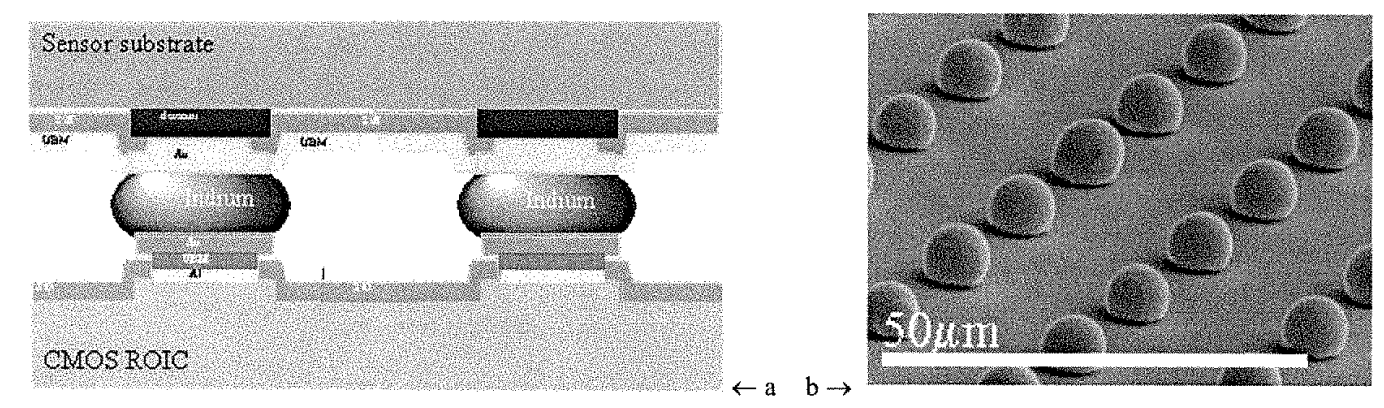

Figure 2. La solution hybride permet de réaliser un détecteur imageur qui tire parti des propriétés exceptionnelles des matériaux à large bande interdite, sans attendre qu'une filière électronique nitrure ou diamant soit disponible. Le circuit de lecture est un dispositif CMOS interfacé par des billes d'indium à la membrane photo-sensible. 


\section{RESULTATS}
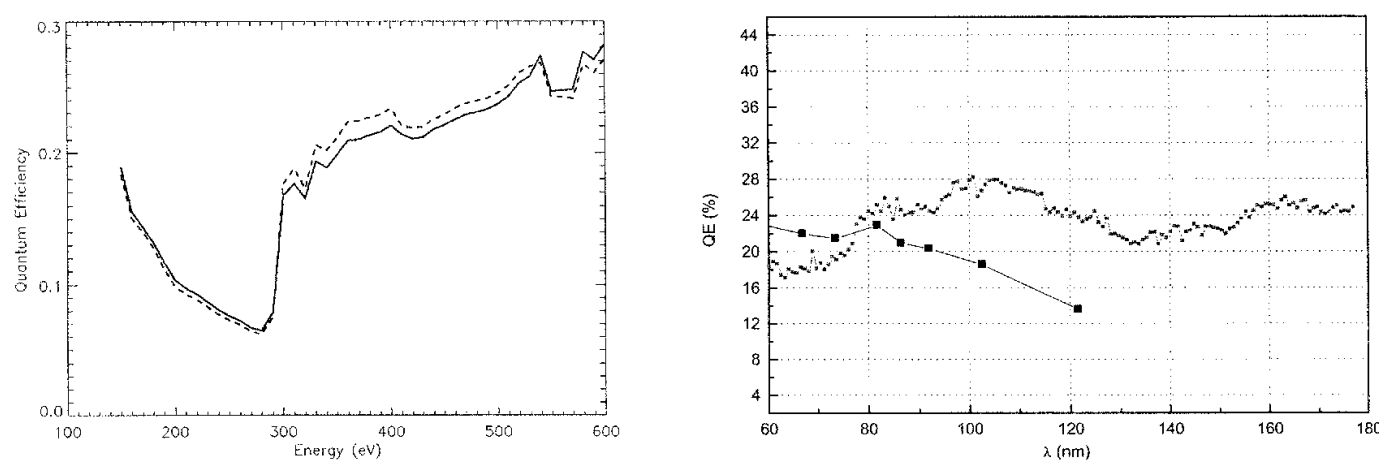

Figure 3. a/ Rendement quantique d'un photoconducteur diamant fabriqué par le LETI, et testé à Super-Aco (Orsay) dans l'XUV [5]. La modification de sensibilité au seuil du carbone est nette. Les 2 courbes correspondent à des polarisations inverses. b/ Rendement quantique d'une diode GaN fabriquée par CHREA (Valbonne) et UPM (Madrid), et testée à Super-Aco

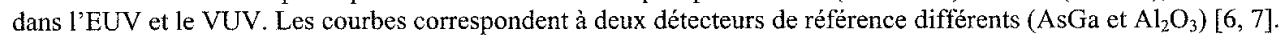

Plusieurs campagnes de mesures $[4,5,6,7,8]$ ont déjà fourni des résultats dont cette section rend brièvement compte. Les mesures se classent en fonction de la gamme de longueur d'onde (XUV, EUV-VUV, NUV-VIS) et selon le matériau étudié (nitrure ou diamant). Les évaluations dans 1'UV lointain se firent sur des lignes de lumière du synchrotron Super-Aco du LURE à Orsay. Une campagne aura lieu fin 2002 au PTB de BESSY à Berlin. Deux résultats prometteurs sont reportés dans les figures 3a (diamant dans l'XUV) et $3 \mathrm{~b}$ ( $\mathrm{GaN}$ dans le VUV). Les mesures dans le proche UV et le visible sont obtenues avec des lampes (LGEP, Gif/Yvette) ou des lasers (LPL, Villetaneuse). Elles démontrent la capacité du diamant et des nitrures à rejeter le visible avec plus de quatre ordres de grandeur [8]. Nous attendons à terme des taux supérieurs par l'amincissement de la membrane photo-sensible, et l'amélioration constatée pour les propriétés optiques des films les plus récents.

\section{CONCLUSION}

Le support financier de l'ESA va permettre de mettre en oeuvre les concepts résultants de plusieurs années d'effort, et de tirer parti de la remarquable expertise présente en BOLD. Nous anticipons dans un proche avenir des détecteurs innovants qui donneront un nouvel essor à l'imagerie UV. Les observations astrophysiques en bénéficieront, mais beaucoup d'autres applications UV devraient profiter de cette avancée. Nous pensons par exemple à l'étude depuis l'espace des changements globaux de la Terre (trou d'ozone) et des aurores boréales, aux procédés lithographiques dont les longueurs d'onde décroissent constamment vers l'extrême UV $(13.5 \mathrm{~nm})$ pour limiter la diffraction, à l'analyse des flammes et des plasmas, aux diagnostiques scientifiques d'instruments tels que le laser X, les lasers UV, les lignes synchrotron, etc. Les résultats seront aussitôt rendus disponibles sur le site bold.oma.be.

\section{Remerciements}

Les auteurs remercient les nombreux collègues qui ont contribué au projet BOLD et l'ont mené à sa forme actuelle. Ils reconnaissent aussi les soutiens financiers du CNES (France) et du Max-Planck-Institut für Aeronomie (Allemagne), le support de l'ESA et des OSTC en Belgique, ainsi que celui de tous les laboratoires et industriels engagés dans le projet.

\section{Références}

[1] Thompson, W. T., UV detectors aboard SOHO, in Proc. SPIE 3764, 196, 1999. 
[2] Hochedez, J.-F., Lemaire, P., Pace, E., Schühle, U., and Verwichte, E., Wide bandgap EUV and VUV imagers for the Solar Orbiter, SP-493 (2001) 245-250, in Proceedings of Solar Encounter, The First Solar Orbiter Workshop, Puerto de la Cruz, Tenerife, Spain, 14-18 May 2001.

[3] Hochedez, J.-F., Schühle, U., Lemaire, P., New UV detector concepts, in The Radiometric Calibration of SoHO, ISSI Scientific Report Series, in press, 2002.

[4] Hochedez, J.-F., Alvarez, J., Auret, F.D., Bergonzo, P., Castex, M.-C., Deneuville, A., Defise, J.M., Fleck, B., Gibart, P., Goodman, S.A., Hainaut, O., Kleider, J.-P., Lemaire, P., Manca, J., Monroy, E., Muñoz, E., Muret, P., Nesladek, M., Omnes, F., Pace, E., Pau, J.L., Ralchenko, V., Roggen, J., Schühle, U., Van Hoof, C., Recent progresses of the BOLD investigation towards UV detectors for the ESA Solar Orbiter, Diamond and Related Materials, 11 (2002) 427-432

[5] Hochedez, J.-F., Verwichte, E., Bergonzo, P., Guizard, B., Mer, C., Tromson, D., Sacchi, M., Dhez, P., Hainaut, O., Lemaire, P., Vial, J.-C., Future diamond UV imagers for solar physics, Physica Status Solidi Applied Research 181, 141, 2000.

[6] Hainaut, O., personal communication, 2002.

[7] Pau, J.L., personal communication, 2002.

[8] Hochedez, J.-F., Bergonzo, P., Castex, M.-C., Dhez, P., Hainaut, O., Sacchi, M., Alvarez, J., Boyer, H., Deneuville, A., Gibart, P., Guizard, B., Kleider, J.-P., Lemaire, P., Mer, C., Monroy, E., Muñoz, E., Muret, P., Omnes, F., Pau, J.L., Ralchenko, V., Tromson, D., Verwichte, E., and Vial, J.-C., Diamond UV detectors for future solar physics missions, Diamond and Related Materials 10 (2001) 673-680 